\title{
Patent Foramen Ovale: Faces of Paradoxical Embolism
}

\author{
Andrew C. Chatzis ${ }^{\mathrm{a}, \mathrm{c}}$, Michael Tsibinos ${ }^{\mathrm{a}}$, Constantinos Xenakis ${ }^{\mathrm{b}}$, Kyriakos Lazaridis $^{\mathrm{b}}$
}

\begin{abstract}
The case of a 73-year-old woman with impending paradoxical embolism is presented. The patient was hospitalised for deep venous thrombosis and transient ischaemic attack. Thorough clinical and laboratory investigation revealed an intracardiac thrombus astride the interatrial septum, most probably originating from the deep veins of the lower extremities. The thrombus was successfully removed surgically.
\end{abstract}

Keywords: Cardiac; Foramen ovale; Paradoxical embolism

\section{Introduction}

A significant number of cerebrovascular accidents are of unknown origin (cryptogenic). It has been suggested that a substantial subgroup of these patients have in fact paradoxical embolism (PDE) as a result of deep venous thrombosis (DVT) in the presence of a patent foramen ovale (PFO). This theory has been strongly criticized partially due to the scarce documentation of impending paradoxical embolism (IPDE) in which a venous thrombus extends from the right atrium through a PFO into the left atrium.

\section{Case Report}

A 73-year-old woman with chronic atrial fibrillation, pre-

\footnotetext{
Manuscript accepted for publication July 9, 2013

${ }^{a}$ Department of Paediatric and Congenital Cardiac Surgery, Onassis Cardiac Surgery Centre, Athens, Greece

${ }^{\mathrm{b}}$ Department of Cardiology, Veterans Hospital, Athens, Greece

${ }^{\mathrm{c} C}$ Corresponding author: Andrew C. Chatzis, Department of Paediatric and Congenital Cardiac Surgery, Onassis Cardiac Surgery Centre, 356 Sygrou Ave, 17674 Kallithea, Athens, Greece.

Email: achatzis@ath.forthnet.gr

doi: http://dx.doi.org/10.4021/jmc1423e
}

sented with DVT of the right lower limb and transient ischaemic attack. Echocardiography revealed the presence of a large, longitudinal, thrombus crossing the PFO, protruding into the left atrium and through the mitral valve into the left ventricle during diastole (Fig. 1A, B).

The patient underwent emergency surgery. At operation under cardiopulmonary bypass and cardiac arrest, right atriotomy was performed. A $2-3 \mathrm{~cm}$ long thrombus was protruding through the PFO (Fig. 2A). The foramen was carefully enlarged and the entire thrombus, $15 \mathrm{~cm}$ long, delivered through. Its shape and size indicated the lower extremities veins as the most probable source (Fig. $2 \mathrm{~B})\left(\mathrm{T}_{1}\right)$. In addition, apparently due to the long standing atrial fibrillation, old wall thrombus was found in the left atrium. This was inadvertently detached from the atrial wall and therefore peeled off and completely removed (Fig. 2B) $\left(\mathrm{T}_{2}\right)$. Fragments of thrombi were looked out for, picked up and removed (Fig. 2B) $\left(\mathrm{T}_{3}\right)$. The left atrial appendage was ligated from the inside and the PFO primarily closed with a double layer suture. The patient made a full recovery and remains well 4 years later on anticoagulation, without further embolic episodes.

\section{Discussion}

The association of PFO and ischaemic stroke is strongly debated [1]. Investigators suggest, based on the high incidence of PFO and the fact that as many as $50 \%$ of DVT cases remain undiagnosed, that a large number of cryptogenic strokes might be attributed to thrombi crossing from the venous to the systemic circulation via a PFO. This is particularly true in young individuals and may account for as many as 47,000 cases a year in the United States alone [2,3]. Furthermore, paradoxical embolism may be responsible for a significant minority of acute peripheral arterial occlusions in the absence of a clear cardiac or proximal arterial source [4].

Impending paradoxical embolism remains the hard evidence of the association of PFO and arterial embolism, yet it is difficult to document. The diagnosis of IPDE has been termed definitive when made at autopsy or in the rare instance when thrombus is seen crossing an intracardiac defect during echocardiography in the face of an arterial embolus 


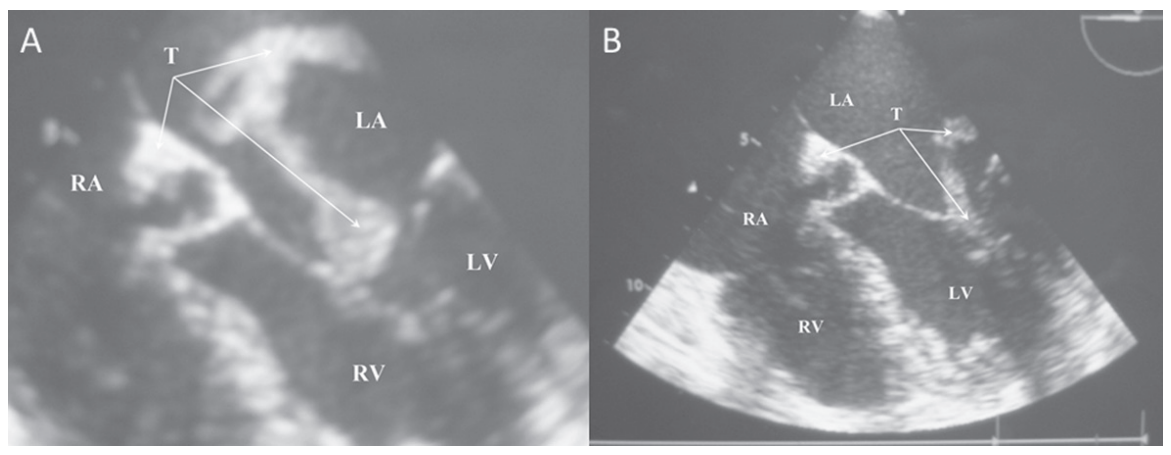

Figure 1. Impending paradoxical embolism. A). Thrombus ( $T$ ) crossing from the right to the left atrium via a patent foramen ovale. B). The same thrombus dropping into the left ventricle during diastole.

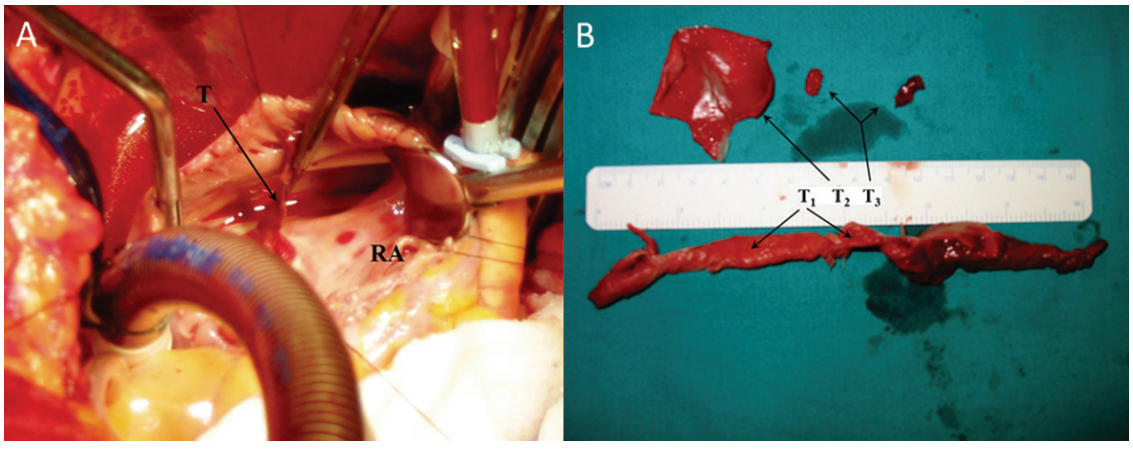

Figure 2. A). Surgeon's view of the thrombus protruding into the right atrium. B). Thrombi removed.

$[2,5]$. We herein present a case of IPDE supported by echocardiographic and surgical evidence where the causative factor was actually "caught in the action".

Consequently it has been suggested that the diagnosis of PDE could be established, should the following 4 criteria were fulfilled: 1). presence of DVT, or pulmonary embolism; 2). abnormal communication between the right (venous) and left (systemic) circulations; 3). clinical, angiographic, or pathologic evidence for systemic embolism, and 4) presence of a favourable pressure gradient promoting right to left shunting [2]. In line with these guidelines several reports have shown up in the literature [4]. Conversely, however, controlled studies failed so far to demonstrate the hypothesised association while PFO closure studies did not show a clear benefit $[1,3]$.

In the rare occasion of an IPDE urgent surgical intervention is mandatory [5]. Currently, secondary stroke prevention after initial ischemic event in young patients with a PFO and atrial septal aneurysm should be based on medical treatment. However, when encountering a young patient with recurrent ischemic stroke, established significant right to left shunt, presence of atrial septal aneurysm and lack of other risk fac- tors, a decision for PFO closure should be considered [3].

\section{References}

1. Davis D, Gregson J, Willeit P, Stephan B, Al-Shahi Salman R, Brayne C. Patent foramen ovale, ischemic stroke and migraine: systematic review and stratified meta-analysis of association studies. Neuroepidemiology. 2013;40(1):56-67.

2. Loscalzo J. Paradoxical embolism: clinical presentation, diagnostic strategies, and therapeutic options. Am Heart J. 1986;112(1):141-145.

3. Piechowski-Jozwiak B, Bogousslavsky J. Stroke and patent foramen ovale in young individuals. Eur Neurol. 2013;69(2):108-117.

4. Travis JA, Fuller SB, Ligush J, Jr., Plonk GW, Jr., Geary RL, Hansen KJ. Diagnosis and treatment of paradoxical embolus. J Vasc Surg. 2001;34(5):860-865.

5. Meacham RR, 3rd, Headley AS, Bronze MS, Lewis JB, Rester MM. Impending paradoxical embolism. Arch Intern Med. 1998;158(5):438-448. 